\title{
A Unified Route Optimization Scheme for Network Mobility ${ }^{\star}$
}

\author{
Jongkeun $\mathrm{Na}^{1}$, Jaehyuk Choi ${ }^{1}$, Seongho $\mathrm{Cho}^{1}$, Chongkwon $\mathrm{Kim}^{1}$, \\ Sungjin Lee ${ }^{2}$, Hyunjeong $\mathrm{Kang}^{2}$, and Changhoi $\mathrm{Koo}^{2}$ \\ 1 School of Electrical Engineering and Computer Science, \\ Seoul National University, Seoul, Republic of Korea \\ $\{j k n a, j h c h o i$, shcho, ckim\}@popeye.snu.ac.kr \\ 2 Telecomunication R\&D Center, \\ Samsung Electronics, Suwon, Republic of Korea \\ \{steve.lee, hyunjeong.kang, chkoo\}@samsung.com
}

\begin{abstract}
Recently Network Mobility(NEMO) is being concerned as new mobility issue. Lots of NEMO issues are already being touched in IETF NEMO WG but the solution is still premature especially to the Route Optimization (RO). NEMO has several problem spaces that need $\mathrm{RO}$ such as nested tunnels problem. Unfortunately, there is no solution that can be universally applied as one for all that results in supporting the coherent network mobility. In this paper, we propose a unified route optimization scheme that can solve several types of RO problem by using Path Control Header (PCH). In our scheme, Home Agent (HA) does piggyback the $\mathrm{PCH}$ on the packet which is reversely forwarded from Mobile Router (MR). That enables any $\mathrm{PCH}$-aware routing facility on the route to make a $\mathrm{RO}$ tunnel with $\mathrm{MR}$ using the Care-of address of MR contained in the PCH. By applying to some already known NEMO RO problems, we show that our scheme can incrementally optimize the routes via default HA-MR tunnel through the simple PCH interpretation.
\end{abstract}

\section{Introduction}

Along with the proliferation of mobile communication networks such as wireless LAN (Local Area Network), PAN (Personal Area Network) and CAN (Car Area Network), most of public transportation systems (e.g. bus, train, airplane) are envisioned to have a permanent connectivity to the Internet even while moving around. In these communication environments, the new mobility problem occurs due to the mobile networks in which the network itself is moving entirely. By now, the literature has only considered how to support host mobility. However, we have to also consider about network mobility because lots of small or medium sized networks require the mobile behavior as same as the current mobile node has in the coming future.

^ This work was supported by Samsung Electronics and the Brain Korea 21 Project. 
The existing Mobile IP [2] solution cannot provide the network mobility because it has different characteristics in comparison with the problem of supporting host mobility. With the need of new protocol for network mobility, a basic protocol was proposed in [1]. It supports transparent mobility to every node in mobile network by using a bi-directional tunnel between the Mobile Router (MR) and the Home Agent (HA). As a protocol extending Mobile IPv6, the MR registers its network prefix as well as its Care-Of Address (CoA) through the extended binding update (a.k.a Prefix Scoped Binding Update [6]) so that HA can properly intercept and tunnel the packets whose destination address belongs to the mobile network prefix to MR's CoA. Basically, this basic protocol can be accepted as a complete network mobility support protocol if we can ignore the routing inefficiency inherently inherited by the bi-directional tunneling. However, the routing efficiency could be more important metric in supporting network mobility than in host mobility. A mobile network which consists of several nodes can consume more link bandwidth and routing resource along the path via $\mathrm{HA}$ in IP routing infrastructure than a single mobile node. Therefore, the route optimization for the efficient IP routing must be considered in this literature along with NEtwork MObility (NEMO) basic protocol [7].

There are some efforts for Route Optimization (RO). For RO in IP routing infrastructure, Some approaches such as [3-5] require a special router or the extension of the existing router which can handle the packet redirection to gain RO effect. The RO schemes belong to this category can be applied to both Mobile IP and NEMO in IP routing infrastructure. On the other hand, There are other kinds of the NEMO-specific RO problem. [8] well defines RO problem spaces of NEMO and briefly analyzes the proposed interim solutions such as $[9$, 10]. Typically, one of NEMO-specific RO problem is a nested tunnels problem that can be formed due to the network mobility. Most of proposed solutions are for solving that problem. As of now, it's not easy to say how RO problems in NEMO can be best solved in the reasonable manner. However, the sure thing is that current proposed solutions can be applied only to one problem space of RO. That is an uncomfortable and unnatural facet in supporting coherent network mobility. We need a simple and effective, unified route optimization solution for network mobility.

In this paper, we propose a route optimization scheme based on Path Control Header $(\mathrm{PCH})$ piggybacking by HA. The scheme is a unified solution that can solve several types of route optimization problem with applying the same principle to the routing facilities such as HA, MR and Correspondent Router (CR), e.g. ORC router in [5]. In the proposed scheme, HA does piggyback the PCH on the packet which is reversely forwarded from MR through the bi-directional MRHA tunnel. PCH is a hop-by-hop option header so that it can be processed by all of the routing facilities on the path that is from HA to Correspondent Node (CN). HA forwards the $\mathrm{PCH}$ piggybacked packets toward $\mathrm{CN}$ for the route optimization. $\mathrm{CR}$ on the path can make a RO tunnel with MR using the information like the CoA of MR contained in the PCH.

Our proposed RO scheme, PCH piggybacking by HA, is a simple and effective one in solving the problems of route optimization without any incompatibility 


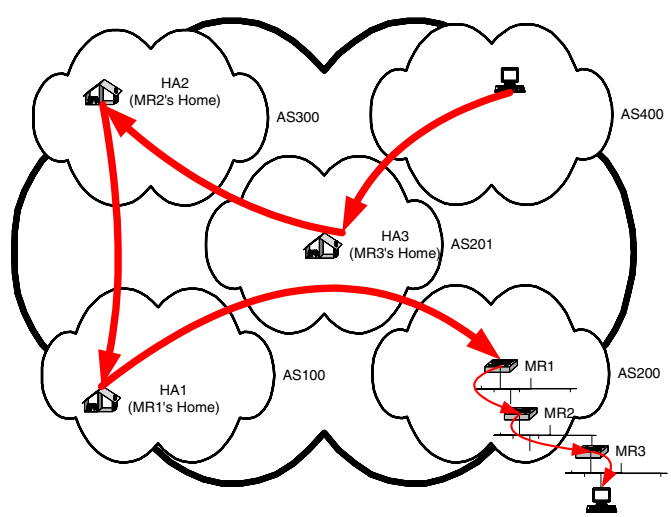

Fig. 1. Nested tunnels optimization in nested mobile networks

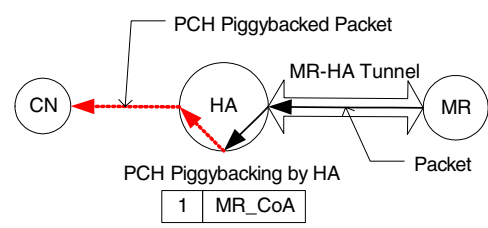

Fig. 2. PCH piggybacking by HA

with the basic NEMO protocol [7]. By taking the functional extension of routing facilities such as HA, MR and CR, we can incrementally optimize the routes over CN-HA-MR without the loss of transparency to CN. And also, we expect that the basic concept of this scheme can be used to support other mobility-related route optimizations as a unified solution, not limited to network mobility.

The rest of this paper is organized as follows: the related works is mentioned in Section 2. In Section 3, we describes the basic operation of PCH based RO scheme. Then, we show how to apply our scheme on RO problem spaces in Section 4. Finally, we conclude in Section 5.

\section{Related Work}

There are some types of route optimization problems. In particular, we can summarize two problems related to NEMO. One is to the route optimization in IP routing infrastructure. The other is to nested tunnels optimization in nested mobile networks. In the former case, CR based approach was introduced in [5]. In there, CR provides the same service to all of CN behind it as if MR supports the transparent mobility service to nodes behind it. This approach can reduce the overhead of applying Mobile IPv6 route optimization to each CN because all of $\mathrm{CN}$ behind $\mathrm{CR}$ can share the optimized tunnel, i.e. RO tunnel, between MR and CR. Also, for the packets reversely forwarded from MR to any CN behind CR, they can be passed to the optimized tunnel, not to the default tunnel between $\mathrm{MR}$ and HA.

In the latter case, it is another type of route optimization problem in NEMO. If multiple mobile networks are nested as Fig.1, that brings a routing overhead to us which is well known as "pinball" or "dog-leg" routing (for the details, see [8]). The packets sent from CN to LFN (Local Fixed Node) get follow the routing path like $\mathrm{CN} \rightarrow \mathrm{HA} 3 \rightarrow \mathrm{HA} 2 \rightarrow \mathrm{HA} 1 \rightarrow \mathrm{MR} 1 \rightarrow \mathrm{MR} 2 \rightarrow \mathrm{MR} 3 \rightarrow \mathrm{LFN}$ by IP routing 


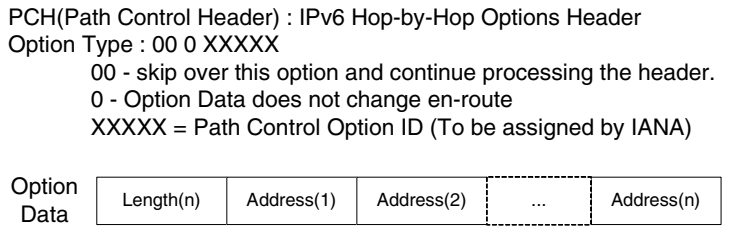

Fig. 3. PCH option format

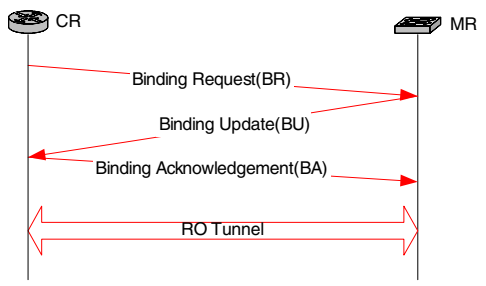

Fig. 4. The signaling procedure for RO Tunnel

and the basic NEMO protocol. In NEMO context, we need to avoid the nested tunneling like this because it incurs very inefficient routing depending on the relative location of HAs. Currently several interim solutions are being proposed in [1] that conceptually convert nested tunnels into a flat tunnel. For the details of NEMO, problem statements and related work can be referred in [1,7-10].

As a result of looking into the route optimization for NEMO, first, we need to devise a solution to allow the route optimization without the limitations such as CN-aware, not scalable, load imbalanced, insecure. Second, we need a unified route optimization scheme that can solve most of problem types related to NEMO.

\section{PCH (Path Control Header) Scheme}

In this section, we introduce the basic concept and operation of our proposed RO scheme in NEMO context.

\subsection{PCH Piggybacking by HA}

To route optimization, $\mathrm{HA}$ does piggyback $\mathrm{PCH}$ on the packet which is reversely forwarded from MR through a bi-directional MR-HA tunnel. PCH is a hop-byhop option header so that it can be processed by all of the routing facilities on the path that is from HA to $\mathrm{CN}$. The mentioned routing facility means an entity which can play a role of the transparent routing agent that can support the packet redirection service like HA. The router in the Internet that implements such an agent function provides the packet redirection service to the nodes behind it by intercepting the packets sent from them and redirecting to the RO tunnel. We call it a CR in here if it is functioning for CNs. The RO tunnel between $\mathrm{CR}$ and MR can be established when $\mathrm{CR}$ gets know the existence of $\mathrm{HA}$ by processing the packet with $\mathrm{PCH}$.

In Fig.2, HA de-capsulates the encapsulated packet forwarded from MR via MR-HA tunnel and then forwards the $\mathrm{PCH}$ piggybacked packet to $\mathrm{CN}$ for the route optimization. Any existing $\mathrm{CR}$ on the path from $\mathrm{HA}$ to $\mathrm{CN}$ can catch the path control information as examining $\mathrm{PCH}$ in the packet. Therefore, the 


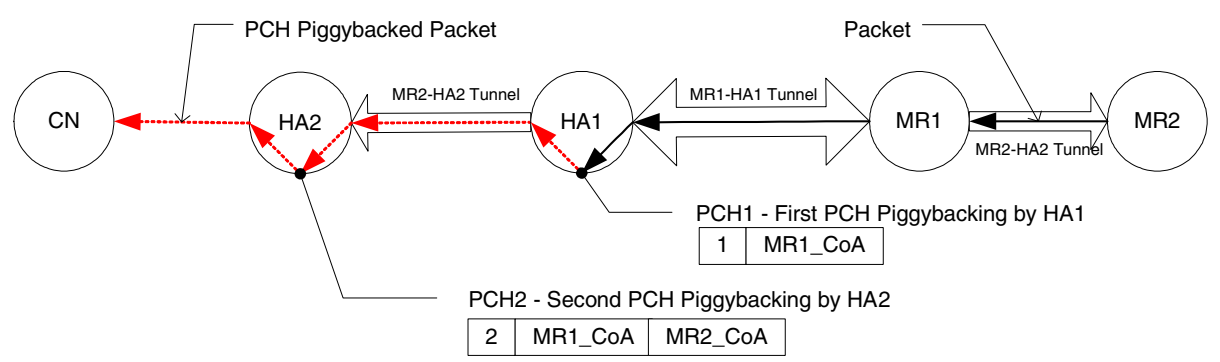

Fig. 5. Nested PCH piggybacking by HAs

CR can initiate the procedure of making a RO tunnel between itself and MR using MR's CoA which is contained in PCH. After setting up the RO tunnel, the packets of $\mathrm{CN}$ will be redirected to the $\mathrm{RO}$ tunnel at $\mathrm{CR}$.

This scheme is simple and effective in respect of RO. It only requires a little effort of HA to provide the RO tunnel between $\mathrm{CR}$ and MR. HA does PCH piggybacking on the packet which is following a non-optimized path of MR-HA tunnel. In here, we can say that CR may be an access router that providing the routing service for a few of subnets or a border router that runs BGP routing protocol in one AS [8].

Fig.3 shows the structure of $\mathrm{PCH}$. $\mathrm{PCH}$ includes an address information as an option data. In here, the address information represents the list of IPv6 addresses. The address contained in $\mathrm{PCH}$ indicates the CoA of MR in MR-HA relationship. Through $\mathrm{PCH}, \mathrm{CR}$ gets know the $\mathrm{CoA}$ of $\mathrm{MR}$ so that $\mathrm{CR}$ can initiate the signaling for RO tunnel.

In Fig.5 that shows the case of forming nested tunnels, $\mathrm{PCH}$ gets contain two CoAs, each of MR1 and MR2. HA2 gets to know the fact that its MR2-HA2 tunnel is nested under the outer MR1-HA1 tunnel after taking a look at the packet with PCH1. The nested HA just adds the CoA of its MR on the received $\mathrm{PCH}$ to make its $\mathrm{PCH}$. Then, $\mathrm{HA} 2$ does piggyback $\mathrm{PCH}$ which includes the $\mathrm{CoA}$ of MR1 (i.e. the exit point of the outer tunnel) and the CoA of MR2 (i.e. the exit point of its tunnel). In this case, one $\mathrm{CR}$ on the path between $\mathrm{HA} 2$ and $\mathrm{CN}$ will be able to make RO tunnel with MR2 by using the nested address information carried in $\mathrm{PCH}$.

\subsection{Making a Route Optimization (RO) Tunnel}

The $\mathrm{CR}$ can make a $\mathrm{RO}$ tunnel after getting the piggybacked $\mathrm{PCH}$ from $\mathrm{HA}$. The signaling to construct a RO tunnel between CR and MR is done with 3-way handshake as in Fig.4. The messages defined in here are carried by Mobility Header defined in [2]. We define new message called BR (Binding Request) to notify MR of the need of RO tunnel. BU (Binding Update) and BA (Binding Acknowledgement) are used for the same purpose as defined in [2] and [7]. And 
also, we define two new mobility options : NRP (Nested Routing Path), RNP (Reachable Network Prefixes).

The initiator of the signaling of RO tunnel should add NRP mobility option in BR message to set up the Nested RO Tunnel with the nested MR. NRP option contains the list of addresses that represents the tree topology of nested MRs. That is used for MR to assign the source routing path that is necessary to nested tunnels optimization. The RNP option is used to let the MR know about the network prefixes which are reachable via RO tunnel. By using this prefix information associated with RO tunnel, MR can select the optimized path (i.e. RO tunnel) for the out-going packets. This option should be contained in BA message.

If the 3-way handshaking $\mathrm{RO}$ signaling between $\mathrm{MR}$ and $\mathrm{CR}$ is done with success, the routing table of both includes new entry for directly reachable prefixes via $\mathrm{RO}$ tunnel. By referring that entry, MR can forward the packets to the established RO tunnel because they are destined to the network that is reachable via it. The CR can do the same thing for the prefix of mobile network that is bound through BU from MR. CR intercepts the packets destined to the prefix and redirects them to the $\mathrm{RO}$ tunnel.

\subsection{Extensions}

For route optimization, MR should understand BR message sent from routing facilities such as CR. According to [2], MR must maintain Binding Update List (BU List). In managing BU List, the following information must be maintained additionally to use RO tunnel defined in this proposed solution. The successful establishment of RO tunnel allows the ready of RO-enabled tunnel interface that would be associated with the correspondent entry of BU List. That tunnel interface should be setup to add IPv6 RH0 (Routing Header Type 0) optional header at the encapsulation of tunneled packets if the NROT (Nested Route Optimization Tunnel) flag is set. The reason why it should do will be explained in Section 4.3. And, MR should maintain the RO tunnels in its own context. In other words, MR can tear down less necessary RO tunnels according to its own criterion such as Least Recently Used (LRU) in the case of resource shortage.

For route optimization, HA should maintain the state of $\mathrm{PCH}$ piggybacking for per traffic flow. The traffic flow can be classified by the destination address of the packets. HA does piggyback $\mathrm{PCH}$ on one packet per the traffic flow. The piggybacking state should be managed by the soft-state. The piggybacking state of per traffic flow comes to be set when the first packet is piggybacked and reset when the state timer is expired. HA doesn't need to piggyback PCH on the packets belong the traffic flow while the correspondent piggybacking state is set. The overhead of managing the piggybacking state can be minimized by the careful implementation. 


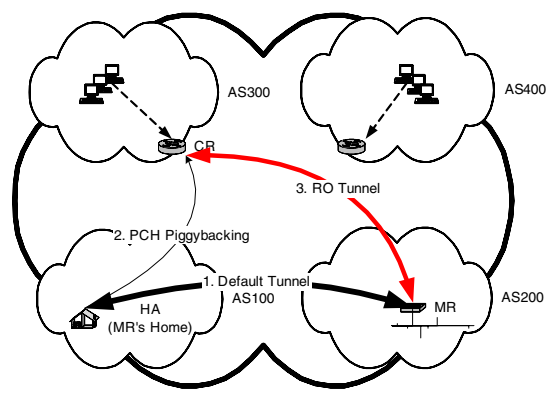

(a) network topology

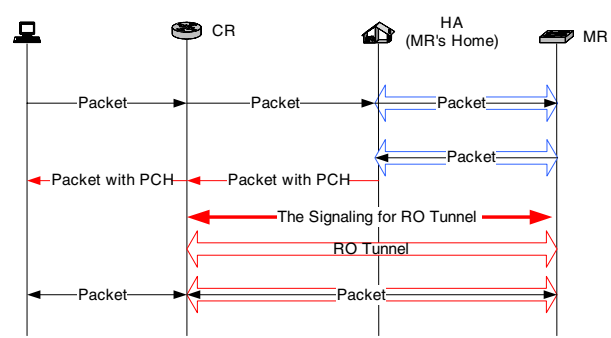

(b) packet sequence

Fig. 6. Route Optimization by single CR

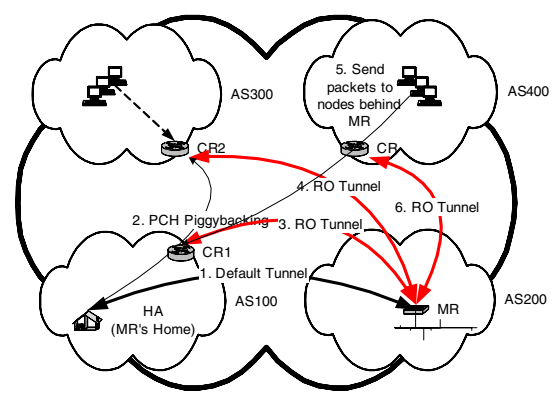

(a) network topology

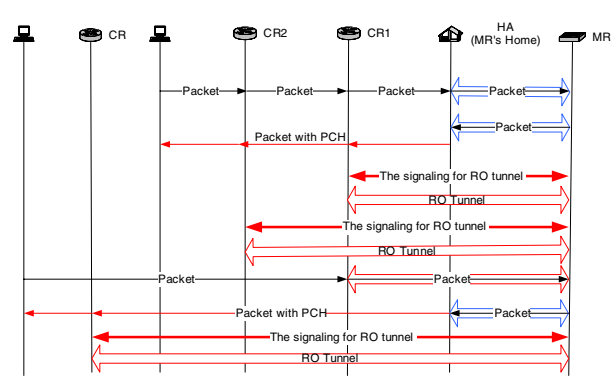

(b) packet sequence

Fig. 7. Route Optimization by multiple CRs

\section{Route Optimization Using $\mathrm{PCH}$}

In this section, we illustrate how to apply our RO scheme on several types of route optimization in NEMO context. For easy understanding, each of route optimization procedures is described together with network configuration and message flow diagram.

\subsection{Route Optimization by CR}

Depending on the location of $\mathrm{CR}$ and how many CRs on the path between $\mathrm{CN}$ and HA, the various types of route optimization can be reflected. In here, we typically show two cases that expose the effect of PCH based RO. First, we assume the network configuration like Fig.6(a). There is one CR on HA-CN path in the border side of AS in which CN exists. In this case, all of other CNs belong the AS can get the gains of route optimization through CR-MR RO tunnel that is pre-established by the $\mathrm{PCH}$ piggybacked packet forwarded from HA to CN. Fig.6(b) is showing the procedure of RO tunnel establishment between $\mathrm{CR}$ and MR. Once established, the real communication between CNs behind CR 


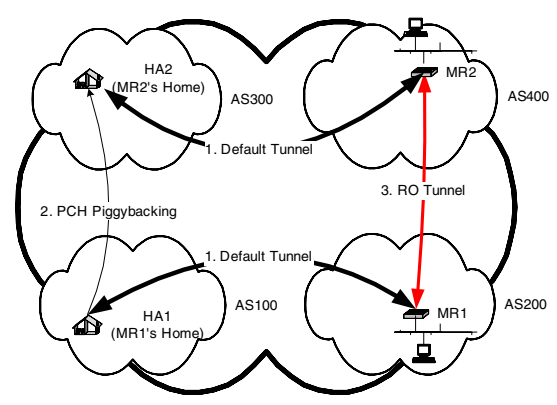

(a) network topology

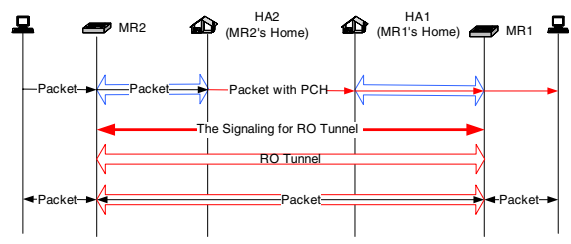

(b) packet sequence

Fig. 8. Route Optimization over MR-to-MR

and nodes behind MR will be realized through the CR-MR RO Tunnel. It is transparent to all of nodes except for CR and MR.

Second, as in Fig.7(a) and Fig.7(b), CR1 and CR2 can simultaneously establish a RO tunnel with MR through one $\mathrm{PCH}$ piggybacking by HA. This is possible because both are on the path that is from HA to CN2. In that case, the packets sent from $\mathrm{CNs}$ in all of subnets attached to CR2 are redirected to RO tunnel at CR2 if they are destined to the mobile network of the MR. CR1 can serve the packets sent from any CNs (in the figure, CN in AS400) that are scattered in the Internet. The packets reached on CR1 indicate that there is no $\mathrm{CR}$ in the path that is from $\mathrm{CN}$ to $\mathrm{CR} 1$, or $\mathrm{CR}$ but still not received $\mathrm{PCH}$. The packets from $\mathrm{CN}$ are redirected at $\mathrm{CR} 1$ and, reversely the packets from $\mathrm{MR}$ are forwarded via HA. At the next time, the $\mathrm{CR}$ on the CR1-CN path can make a $\mathrm{RO}$ tunnel by picking up on $\mathrm{PCH}$ in the reversely forwarded packet from HA. As a result of $\mathrm{PCH}$ piggybacking by $\mathrm{HA}$, we can serve the incremental route optimization to all of CNs.

\subsection{Route Optimization over MR-to-MR}

As in Fig.8(a) and Fig.8(b), we can get the RO tunnel over MR-to-MR by using $\mathrm{PCH}$ piggybacking. MR per se interprets $\mathrm{PCH}$ piggybacked from the HA of the other MR and initiates the signaling for RO tunnel with the other MR. As a result of that, the nodes behind one MR can directly communicate with the nodes behind the other MR without any routing overhead.

\subsection{Nested Tunnels Optimization (NTO)}

Our scheme can also be applied to solve the nested tunnels problem without the loss of generality. We assume the 3-level nested network configuration as Fig.9(a) to show NTO using PCH based scheme. In nested mobile networks, the RO tunnel is called NROT (Nested RO Tunnel) because we introduce the source routing concept in handling the nested tunnels optimization. To the correct 


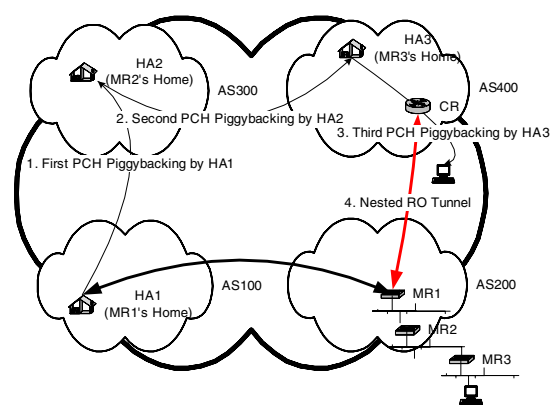

(a) network topology

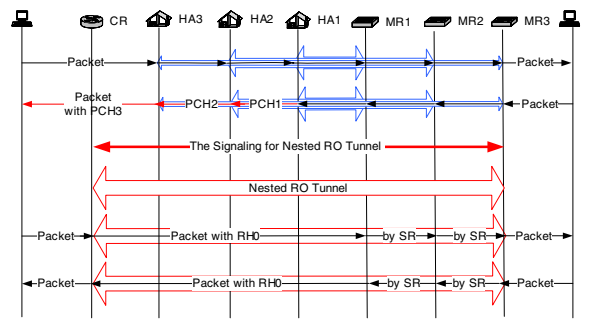

(b) packet sequence

Fig. 9. Nested Tunnels Optimization by CR

\begin{tabular}{|c|c|c|c|c|c|}
\hline \multirow[t]{2}{*}{$\mathrm{CR}$} & \multirow[t]{2}{*}{ MR1: } & MR1_CoA & CR_Addr & 2:MR2_CoA | MR3_CoA & $\begin{array}{l}\text { Original Pkt. } \\
\text { from } \mathrm{CN}\end{array}$ \\
\hline & & \multicolumn{2}{|l|}{ DST } & $\mathrm{RHO}$ & \\
\hline \multirow[t]{2}{*}{ MR1 } & \multirow[t]{2}{*}{ MR2: } & MR2_CoA & CR_Addr & 1:MR1|MR3_CoA & $\begin{array}{l}\text { Original Pkt. } \\
\text { from CN }\end{array}$ \\
\hline & & DST & SRC & RHO & \\
\hline \multirow[t]{2}{*}{ MR2 } & \multirow[t]{2}{*}{ MR3: } & MR3_CoA & CR_Addr & $0:$ MR1 | MR2 & $\begin{array}{l}\text { Original Pkt. } \\
\text { from CN }\end{array}$ \\
\hline & & DST & SRC & $\mathrm{RHO}$ & \\
\hline
\end{tabular}

(a) forward direction

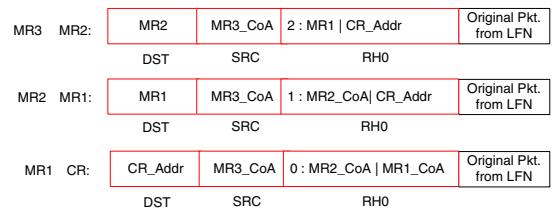

(b) backward direction

Fig. 10. Packet Delivery via Nested Route Optimization Tunnel

routing in the nested network configuration, we take advantage of IPv6 Routing Header Type 0 (RH0) in NROT.

In Fig.9(a), CR gets to know the existence of nested tunnels through PCH information (MR1's CoA and MR2's CoA, MR3's CoA) and then initiate the signaling for NROT to MR3 via nested tunnels. At this time, the Binding Request (BR) message contains the NRP Option. The NRP Option is used to inform MR3 of the nested path information. If MR3 receives the BR message having the NRP option, MR3 also gets know that it is nested. Therefore, the tunnel between CR and MR3 becomes a NROT.

In a NROT, the entry point of tunnel adds RH0 at encapsulation. Reversely, the exit point of tunnel deletes RH0 at decapsulation. For the packets tunneled from CR to MR3, the packet forwarding is done with source routing of RH0 $(\mathrm{MR} 1 \rightarrow \mathrm{MR} 2 \rightarrow \mathrm{MR} 3)$. For the packets tunneled from MR3 to CR, the reverse source routing $(\mathrm{MR} 2 \rightarrow \mathrm{MR} 1 \rightarrow \mathrm{CR}$ ) occurs. Fig.9(b) shows message flow for NTO by CR. Fig.10(a) and Fig.10(b) show the content of RH0 packet at the packet delivery via NROT.

\section{Conclusion and Future Work}

As a unified solution for NEMO RO, we introduced the concept and basic operations of our proposed scheme which implemented by PCH Piggybacking in the HA. We proved that the proposed scheme can be used to solve most of the RO 
problems defined in [8] as a unified solution by showing the RO cases based on $\mathrm{PCH}$ in Section 4. We expect that the basic concept of our scheme can be used to support other mobility-related route optimizations as a unified solution, not limited to NEMO.

As a next step, we are going to evaluate our proposed scheme through simulation and experiment to answer the following questions. 1) What is the signaling overhead compared to the gains of route optimization? 2) How well does our scheme under the various handover scenarios? 3) How much can we get the throughput gain through applying RO? And, can that fully compensate the cost of installing CRs in the Internet? And also, we need to quantitatively compare our scheme with other approaches being proposed in IETF NEMO WG or other research area. Lastly, we also leave the detailed security consideration into the future work.

\section{References}

1. NEtwork MObility: NEMO web page, http://www.ietf.org/html.charters/nemocharter.html, Jan. 2004.

2. C. Perkins, D. Johnson and J. Arkko, "Mobility Support in IPv6," draft-ietfmobileip-pv6-18 (work in progress), July 2002.

3. Fumio Teraoka, Keisuke Uehara, Hideki Sunahara, and Jun Murai, "VIP : A Protocol Providing Host Mobility," Aug. 1994

4. Weidong Chen, Eric Lin, "Route Optimization and Location Updates for Mobile Hosts," International Conference on Distributed Computing Systems, 1996

5. Ryuji Wakikawa, Susumu Koshiba, Keisuke Uehara, Jun Murai, "ORC: Optimized Route Cache Management Protocol for Network Mobility," Proc. of ICT2003, Nov 2003.

6. Ernst, T., Castelluccia, C., Bellier, L., Lach, H. and A. Olivereau, "Mobile Networks Support in Mobile IPv6 (Prefix Scope Binding Updates)," IETF draft-ernstmobileip-v6-network-03 (work in progress), March 2002.

7. Vijay, D., Ryuji, W., Alexandru, P., Pascal, T., "NEMO Basic Support Protocol," IETF draft-ietf-nemo-basic-support-02(work in process), December 2003.

8. Thubert, P., and Molteni, M., "Taxonomy of Route Optimization Models in the NEMO Context," IETF draft-thubert-nemo-ro-taxonomy-02 (work in progress), February 2004.

9. Thubert, P., and Molteni, M., "IPv6 Reverse Routing Header and Its Application to Mobile Networks," IETF draft-thubert-nemo-reverse-routing-header-04 (work in progress), February 2004.

10. Chan-Wah Ng, and Takeshi Tanaka, "Securing Nested Tunnels Optimization with Access Router Option," IETF draft-ng-nemo-access-router-option-00 (work in progress), Oct. 2002. 